\begin{abstract}
Iranica
Abstracta Iranica Revue bibliographique pour le domaine irano-aryen

Volume 42-43 | 2021

Comptes rendus des publications de 2019-2020
\end{abstract}

\title{
Yossef Rapoport. Islamic Maps
}

\section{Francesco Calzolaio}

\section{(2) OpenEdition}

\section{Journals}

Édition électronique

URL : https://journals.openedition.org/abstractairanica/53704

DOI : 10.4000/abstractairanica.53704

ISSN : 1961-960X

Éditeur :

CNRS (UMR 7528 Mondes iraniens et indiens), Éditions de l'IFRI

\section{Référence électronique}

Francesco Calzolaio, «Yossef Rapoport. Islamic Maps », Abstracta Iranica [En ligne], Volume 42-43 | 2021, document 18, mis en ligne le 30 décembre 2021, consulté le 24 décembre 2022. URL : http:// journals.openedition.org/abstractairanica/53704 ; DOI : https://doi.org/10.4000/abstractairanica. 53704

Ce document a été généré automatiquement le 24 décembre 2022.

Tous droits réservés 


\title{
Yossef Rapoport. Islamic Maps
}

\author{
Francesco Calzolaio
}

\section{RÉFÉRENCE}

Yossef Rapoport. Islamic Maps. Oxford: Bodleian Library, 2020, 192 p.

1 Le livre, enrichi par soixante illustrations de couleurs d'excellente qualité, propose une histoire générale des pratiques de cartographie dans le monde islamique médiéval et moderne. La discussion se structure sur six chapitres essentiellement chronologiques.

Le premier, "A Mathematicians' Map of the Nile », est centré sur la cartographie mathématique avec une attention particulière portée sur la carte du Nil du géographe

du IXe siècle al-Khwārazmī, dont l'A. souligne la dette par rapport au savoir grec.

Ensuite, l'étude tractation porte sur al-Istakhrī (fl. Xe siècle) et son œuvre, qui font

l'objet du deuxième chapitre. Tandis que al-Khwārazmī est invoqué comme le représentant de la cartographie mathématique, l'œuvre d'al-Istakhrī est en revanche

citée en exemple de cartographie pratique, où la rigueur mathématique et la proportionnalité sont sacrifiées pour des dessins géométriques abstraits proposant une vision iconique de la nature interconnectée du monde islamique. Le troisième chapitre

est consacré au

\section{Livre des curiosités}

, une cosmographie anonyme d'atant du XIe siècle dont l'A. est l'un des principaux spécialistes. Composé sous la dynastie Fatimide, le livre dévoue une attention spéciale à

l'espace méditerranéen, dont il offre une représentation fortement politisée. Le quatrième chapitre porte sur l'œuvre d'al-Idrīsī (m. après 1154), dont l'A. propose une contextualisation historique et résume les caractéristiques fondamentales, avec une attention particulière à sa relation avec le savoir géographique antérieur. Dans le cinquième chapitre, l'A. se concentre en revanche sur la carte du monde du célèbre capitaine de navire et cartographe Pīrī Re'îs (m. 1554) et son 


\section{Livre de Navigation}

pour tracer une histoire des débuts de la cartographie ottomane. Pour conclure, le sixième mène une discussion sur le rapport entre science, pouvoir et religion en Iran Safavide en s'appuyant sur un astrolabe safavide.

Dans la mesure où il vise à offrir au lecteur une vue d'ensemble sur un sujet aussi vaste Islamic Maps que la cartographie islamique en moins de deux-cents pages,

est un livre ambitieux. Le spécialiste en études persanes aurait souhaité davantage d'attention au monde de culture persane. Un chapitre sur l'Empire timouride ou bien moghol, par exemple, aurait permis d'enrichir ultérieurement l'analyse. Dans

l'ensemble, l'expérience atteint pourtant son but. L'A. offre une introduction aux fondements de la cartographie islamique bien écrite et mise à jour en fonction de l'état de la recherche, et la bibliothèque d'images qu'il propose est très riche, pertinente et admirablement réalisée sous l'angle technique.

\section{AUTEURS}

\section{FRANCESCO CALZOLAIO}

Paris 